\title{
Bench-top X-ray microtomography complemented with spatially localized X-ray scattering experiments
}

\section{Suuronen, Jussi-Petteri}

2014

Suuronen , J-P , Kallonen , A P , Hänninen , V , Blomberg , M , Hämäläinen , K \& Serimaa , R 2014 , ' Bench-top X-ray microtomography complemented with spatially localized X-ray scattering experiments ' , Journal of Applied Crystallography , vol. 47 , pp. 471-475 . https://doi.org/10.1107/S16005

http://hdl.handle.net/10138/42767

https://doi.org/10.1107/S1600576713031105

publishedVersion

Downloaded from Helda, University of Helsinki institutional repository.

This is an electronic reprint of the original article.

This reprint may differ from the original in pagination and typographic detail.

Please cite the original version. 


\section{Bench-top X-ray microtomography complemented with spatially localized X-ray scattering experiments}

\section{Jussi-Petteri Suuronen, Aki Kallonen, Ville Hänninen, Merja Blomberg, Keijo Hämäläinen and Ritva Serimaa}

J. Appl. Cryst. (2014). 47, 471-475

Copyright (C) International Union of Crystallography

Author(s) of this paper may load this reprint on their own web site or institutional repository provided that this cover page is retained. Republication of this article or its storage in electronic databases other than as specified above is not permitted without prior permission in writing from the IUCr.

For further information see http://journals.iucr.org/services/authorrights.html

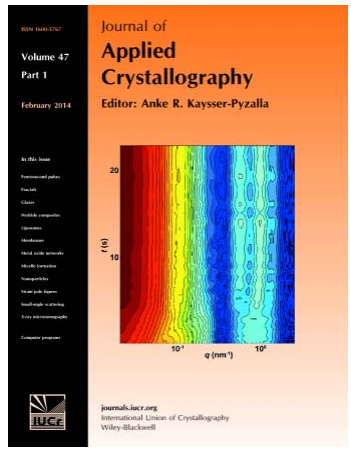

Many research topics in condensed matter research, materials science and the life sciences make use of crystallographic methods to study crystalline and non-crystalline matter with neutrons, X-rays and electrons. Articles published in the Journal of Applied Crystallography focus on these methods and their use in identifying structural and diffusioncontrolled phase transformations, structure-property relationships, structural changes of defects, interfaces and surfaces, etc. Developments of instrumentation and crystallographic apparatus, theory and interpretation, numerical analysis and other related subjects are also covered. The journal is the primary place where crystallographic computer program information is published.

Crystallography Journals Online is available from journals.iucr.org 
Journal of

Applied

Crystallography

ISSN 1600-5767

Received 13 September 2013

Accepted 12 November 2013

\section{Bench-top X-ray microtomography complemented with spatially localized $X$-ray scattering experiments}

\author{
Jussi-Petteri Suuronen, ${ }^{*}$ Aki Kallonen, Ville Hänninen, Merja Blomberg, Keijo \\ Hämäläinen and Ritva Serimaa
}

Department of Physics, University of Helsinki, PO Box 64, Helsinki, 00014, Finland. Correspondence e-mail: jussi-petteri.suuronen@helsinki.fi

This article describes a novel experimental setup that combines X-ray microtomography (XMT) scans with in situ X-ray scattering experiments in a laboratory setting. Combining these two methods allows the characterization of both the micrometre-scale morphology and the crystallographic properties of the sample without removing it from the setup. Precise control of the position of the sample allows an accurate choice of the scattering beam path through the sample and facilitates the performance of X-ray scattering experiments on submillimetre-sized samples. With the present setup, a voxel size of less than $0.5 \mu \mathrm{m}$ is achievable in the XMT images, and scattering experiments can be carried out with a beam size of approximately $200 \times 200 \mu \mathrm{m}$. The potential of this setup is illustrated with the analysis of micrometeorite crystal structure and diffraction tomographic imaging of a silver behenate phantom as example applications.

\section{Introduction}

Since its introduction in the late 1980s (Flannery et al., 1987), X-ray microtomography (XMT) has become a popular and powerful method for nondestructively evaluating material structures (Stock, 2008). When combined with more traditional analysis methods like X-ray diffraction (XRD), small-angle scattering (SAXS) or X-ray fluorescence spectroscopy, it extends the range of phenomena that can be observed with X-ray methods from the atomic to the micrometre scale, bridging the gap between scattering-based or spectroscopic methods and traditional X-ray imaging. Such multimodal studies incorporating both scattering and tomographic information have been applied to a wide range of problems, from biological materials like sea urchin teeth (Stock et al., 2002) or wood samples (e.g. Leppänen et al., 2011; Penttilä et al., 2013) to engineering issues like the effects of sulfate attack on Portland cement (Naik et al., 2005). All of these studies employed a bench-top XMT scanner in conjunction with a dedicated instrument for the scattering measurements.

Previously, the use of synchrotron radiation has allowed the two experiments to be performed sequentially at the same beamline and with higher temporal resolution (e.g. Pyzalla et al., 2005) than is achievable with an X-ray tube source. Also possible with a synchrotron source are various types of diffraction or scattering contrast tomography, where the tomographic reconstruction is formed not on the basis of a projection radiograph, but using some quantity (crystal orientation, crystallinity, $d$ spacing etc.) calculated from a large number of scattering patterns obtained with the pencilbeam (translate/rotate) imaging geometry (Álvarez-Murga et al., 2012; Voltolini et al., 2013). In addition, small-angle scattering contrast in the pencil-beam geometry has been reported (Schroer $e t$ al., 2006). Furthermore, high-brightness synchrotron radiation in combination with novel crystal analyzers enables even more exotic contrast mechanisms using molecular level spectroscopic information (Huotari et al., 2011). Two closely related imaging approaches particularly suited for grain mapping of polycrystalline material specimens are three-dimensional X-ray microscopy (3DXRD) and diffraction contrast tomography (DCT). Both techniques rely on identifying and sorting diffraction and/or extinction spots occurring when individual grains in the sample fulfill the Bragg condition. The 3DXRD method (see e.g. Poulsen, 2012) uses either a line beam or a parallel beam and two area detectors to record the diffraction patterns: a high-resolution detector near the sample yields spatial information on the grains, whereas a low-resolution detector further away is used for structural characterization. In the DCT setup (Ludwig et al., 2008; Johnson et al., 2008; Reischig et al., 2013), only one detector is used, but analysis similar to the 3DXRD method is incorporated with conventional absorption tomography.

While synchrotron-based laboratories will undoubtedly remain at the forefront of method development, incorporating these techniques into smaller-scale systems is crucial for their wider adoption in the scientific community. Recently, first steps have been taken in this direction, when the DCT method was implemented in an X-ray-tubebased setup by King et al. (2013).

In this work, we present a home laboratory setup that implements the pencil-beam approach, using a second X-ray tube and an area detector mounted around a commercial bench-top XMT scanner to complement X-ray microtomography scans with in situ X-ray scattering experiments. On the basis of the XMTreconstruction, a specific sub-volume of the sample can be selected for the scattering experiment with $200 \mu \mathrm{m}$ resolution; this facilitates scattering experiments on submillimetre-sized samples and allows mapping of selected crystallographic properties within larger samples.

\section{Experimental setup}

\subsection{System overview}

The setup, presented in Fig. 1, is constructed around a custom-built high-resolution XMT scanner (Nanotom 180NF, Phoenix|x-ray 
Systems and Services GmbH, presently part of GE Measurement and Control Solutions, Germany), capable of imaging the sample with a detail detectability down to $200 \mathrm{~nm}$. This resolution is comparable to what is achievable at a modern synchrotron microtomography setup (Kastner et al., 2010), although the latter has the advantages of a better signal-to-noise ratio, lack of polychromaticity artifacts and the possibility for phase-contrast imaging. X-ray scattering functionality is provided by an additional molybdenum-anode X-ray source ( $\mathrm{I} \mu \mathrm{S}$, Incoatec $\mathrm{GmbH}$, Germany), and a state of the art Pilatus 1M detector (Dectris Ltd, Switzerland) is used to measure the scattering pattern. Focusing multilayer Montel optics and a variable divergence aperture allow the adjustment of the beam size at the sample to optimize resolution and scattering intensity. The scattering beam is targeted at the desired part of the sample using the computer-controlled sample manipulator stage of the XMT scanner.

\subsection{The XMT system}

The Nanotom 180NF XMT scanner consists of a transmission-type $\mathrm{X}$-ray tube, the sample manipulator stage and a 5 megapixel CMOS flat-panel detector (Hamamatsu Photonics, Japan). To accommodate the additional equipment of the scattering system, the scanner has been custom built inside a $2.8 \times 3.8 \times 2.4 \mathrm{~m}$ lead-shielded room instead of the more conventional radiation shielding cabinet. The CMOS detector has $2304 \times 2304$ pixels, each with an area of $50 \times$ $50 \mu \mathrm{m}$. X-rays are emitted from the tube in a divergent cone beam, and the effective edge length of one pixel in the transmission images, $s_{\mathrm{p}}$, is a function of the imaging geometry:

$$
s_{\mathrm{p}}=\frac{D_{\mathrm{SO}}}{D_{\mathrm{SD}}} 50 \mu \mathrm{m},
$$

where $D_{\mathrm{SO}}$ and $D_{\mathrm{SD}}$ are the distances from the X-ray source to the sample and the detector. The transmission target material is tungsten, and the maximum acceleration voltage for the X-ray tube is $180 \mathrm{kV}$. The reconstructions are computed with the datos $\mid x$ reconstruction software provided by the equipment manufacturer. The software allows the voxel size of the reconstruction to be chosen to be $0.5,1,2$ or 4 times the effective pixel size of the transmission images. In addition to the voxel size, the scan resolution is limited by the size of the X-ray source, i.e. the size of the electron beam hitting the transmission target. This can be selected from four tube mode settings with quoted detail detectability of 200, 330, 650 and $900 \mathrm{~nm}$. A more detailed description of the system and analysis of its performance is given elsewhere (e.g. Brunke et al., 2008).

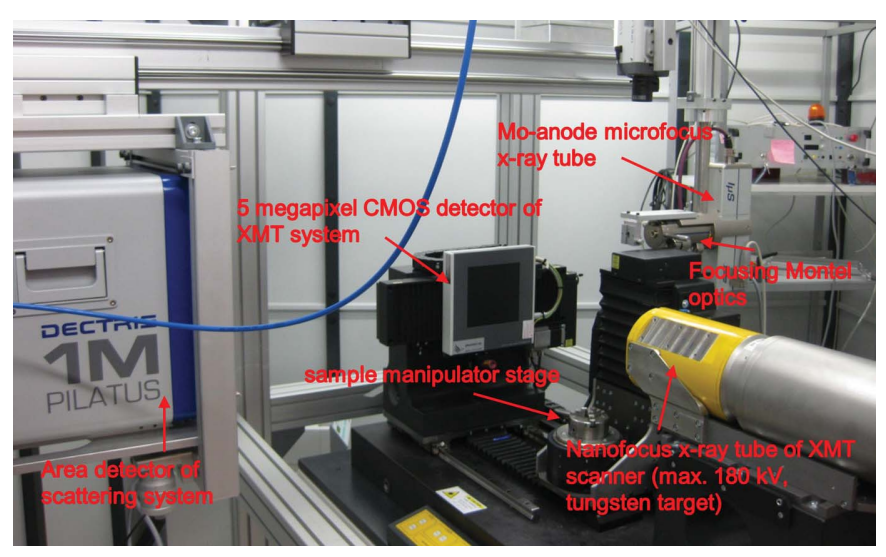

Figure 1

The combined X-ray microtomography and scattering setup.

\subsection{The scattering system}

2.3.1. I $\mu \mathrm{S} X$-ray tube and optics. The X-ray beam for the scattering experiments is provided by the microfocus X-ray tube $\mathrm{I} \mu \mathrm{S}$, with a maximum tube power of $30 \mathrm{~W}(50 \mathrm{kV} \times 600 \mu \mathrm{A})$. The beam is simultaneously focused and monochromated in the Montel optics by reflecting from two perpendicular graded multilayer mirrors. After the optics, there are four beams in the beam path: the focused and monochromatic beam, two diverging beams that have only been reflected by one of the two mirrors, and a white beam that passes through the optics without reflecting. The size of the beams can be adjusted with the variable divergence aperture, which is situated immediately after the optics. To cut off the three undesired beams, an additional vertical slit is mounted $6 \mathrm{~cm}$ after the variable divergence aperture. The rectangular doubly reflected beam is shown in Fig. 2. Molybdenum was chosen for the anode material since the $K \alpha$ radiation is of sufficiently low energy (approximately $17 \mathrm{keV}$ ) to enable studies of soft matter samples like clays with a reasonably good $q$ resolution, while still being energetic enough to eliminate the need for a vacuum in the beam path because of air scattering. On the high-energy side, a limiting factor was the decreased efficiency of the detector at high photon energies.

2.3.2. Pilatus $\mathbf{1 M}$ detector. The scattering pattern is recorded with a Pilatus $1 \mathrm{M}$ hybrid pixel array detector, which has $981 \times 1064$ pixels of $172 \times 172 \mu \mathrm{m}$. The sample-to-detector distance can be varied to suit the desired range of scattering angles, enabling measurements from the wide-angle X-ray scattering (WAXS) regime to moderately small scattering angles. The largest possible sample-to-detector distance is $75 \mathrm{~cm}$, which gives a largest detectable $d$ spacing of approximately $60 \AA$, corresponding to a magnitude of the scattering vector of $0.1 \AA^{-1}$.

\subsection{Beam alignment for WAXS/SAXS measurements}

A key problem in utilizing the setup is correlating the X-ray scattering pattern with the illuminated sub-volume of the sample. This is achieved with a beam position phantom, consisting of a small silver behenate particle $(\sim 200 \mu \mathrm{m}$ in diameter, approximately the same as the scattering beam) mounted on the tip of a steel needle. On the basis of an XMT reconstruction, the exact coordinates of the

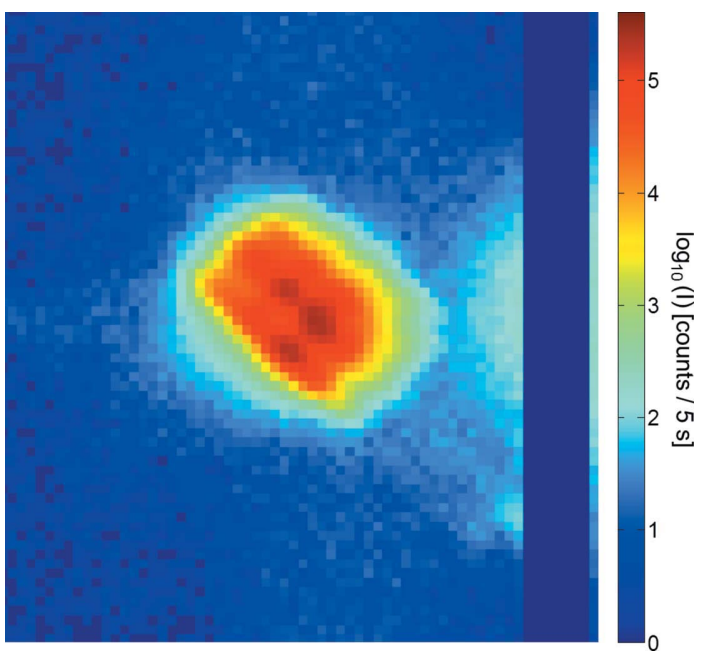

Figure 2

Image of the diffracting beam $75 \mathrm{~cm}$ after the sample, pixel size $172 \times 172 \mu \mathrm{m}$. At the sample, the horizontal width of the beam is approximately $200 \mu \mathrm{m}$. The structure seen in the beam is due to molybdenum $K \alpha_{1}$ and $K \alpha_{2}$ radiation being reflected to slightly different angles from the two mirrors in the optics. The vertical bar on the right is a gap between modules on the modular detector. 
particle are known in the reference frame of the sample manipulator axes; by maximizing the intensity of silver behenate diffraction peaks on the detector, the position of the scattering beam can also be fixed in the same coordinates. Once the location of the scattering beam is known, correct manipulator coordinates for a WAXS/SAXS measurement of a specified sub-volume in any XMT reconstruction can be calculated with some elementary trigonometry.

\section{Example experiments and results}

\subsection{X-ray diffraction tomography}

The resolution of the X-ray scattering system was verified by performing an X-ray diffraction tomography (XDT) scan on a test sample consisting of two particles of silver behenate, both of approximately $300 \mu \mathrm{m}$ in diameter and with approximately $200 \mu \mathrm{m}$ separation between the particles. Firstgeneration (translate-rotate) geometry was used with 12 angular steps from 0 to $165^{\circ}$, and 11 diffraction patterns were obtained with $200 \mu \mathrm{m}$ separation at each projection angle. ${ }^{1}$ The intensity of the silver behenate 001 diffraction peak was determined from each scattering pattern and used as the projection variable for tomographic reconstruction with the standard filtered back-projection algorithm. Prior to reconstruction, the resulting sinogram was oversampled to $21 \times 12$ in order to obtain a smoother-looking reconstruction.

Fig. 3 illustrates this process and shows the resulting XDT reconstruction overlaid on a conventional XMT reconstruction of the same sample. Although the voxel size in the XDT reconstruction is much larger $(100 \times 100 \times 100 \mu \mathrm{m}$ versus $3.8 \times 3.8 \times 3.8 \mu \mathrm{m})$, the two particles are clearly resolved in both reconstructions. It should be noted that, since the particles are relatively small compared to the voxel size, the XDT reconstruction exhibits a significant partial volume effect, and one should not expect perfect agreement between the two. The XMT scan was performed with $60 \mathrm{kV}$ X-ray tube voltage, $100 \mu \mathrm{A}$ current and the focus mode with $900 \mathrm{~nm}$ detail detectability.

\subsection{Micrometeorite crystal structure}

There are several application fields where the combined X-ray scattering and tomography setup provides a significant advantage over having a separate setup dedicated for small- or wide-angle scattering experiments. One such example is in the study of micrometeorites, where XMT results can be used for accurate volume determination and analysis of their morphological characteristics and combined with XRD results to determine their mineralogical composition (Nakamura et al., 2008). In this case, the small size of the samples (200-600 $\mu \mathrm{m}$ in diameter) makes it difficult to position them for an XRD experiment with a dedicated instrument, especially since manual handling of the samples should be kept to a minimum to avoid losing the samples or contaminating them with external

1 This corresponds to an angular range of $180^{\circ}$ with angular step of $15^{\circ}$. The projection at $180^{\circ}$ would contain redundant information as it is a mirror image of the data at $0^{\circ}$. material. The combined setup provides a significant advantage when the two experiments can be performed sequentially without removing the sample from the setup. Using an X-ray-tube-based instrument eliminates the time lag from applying for synchrotron beam time months ahead of the experiment, while the XRD results can still be linked to specific features observed in the XMT reconstructions.

The results of such an experiment on two micrometeorites from the Atacama Desert are presented in Fig. 4. The meteorites were placed at the bottom of sealed polypropylene pipette heads, which were filled with cotton to avoid sample movement during the XMT scan. The XMT scans were carried out with an effective pixel size $s_{\mathrm{p}}=0.5 \mu \mathrm{m}$, an $80 \mathrm{kV}$ X-ray tube voltage and a $180 \mu \mathrm{A}$ current. The largest focus mode was selected, as no blurring of the transmission images was observed in comparison with the finer focus modes. On the basis of the XMT reconstructions (Figs. $4 a$ and $4 c$, voxel size $0.5 \mu \mathrm{m})$, two XRD measurements per sample were carried out over the $q$ range of approximately $0.5-5 \AA^{-1}$, using the 111 diffraction peak from a silicon powder sample to determine the $q$ scale. While XRD experiments revealed both meteorites to be of a primarily olivine composition, there is a significant difference in the degree of crystallite orientation within the samples: the scattering pattern of sample A (Fig. 4b) is indicative of a very well ordered material of high crystallinity, while sample B gives a powder-like diffraction pattern (Fig. 4d). When the XRD beam is directed through the large inclusion within sample $\mathrm{B}$, scattering from the inclusion is seen superimposed on the powder pattern (Fig. 4e). Unfortunately, these reflections only contribute two peaks (at $q=1.864 \AA^{-1}$ and $q=1.961 \AA^{-1}$ ) to the integrated diffraction pattern (Fig. $4 f$ ), making mineral identification very difficult.

\section{Conclusions}

This work describes a unique home laboratory instrument that enables X-ray microtomography scans to be complemented with 


\section{laboratory notes}

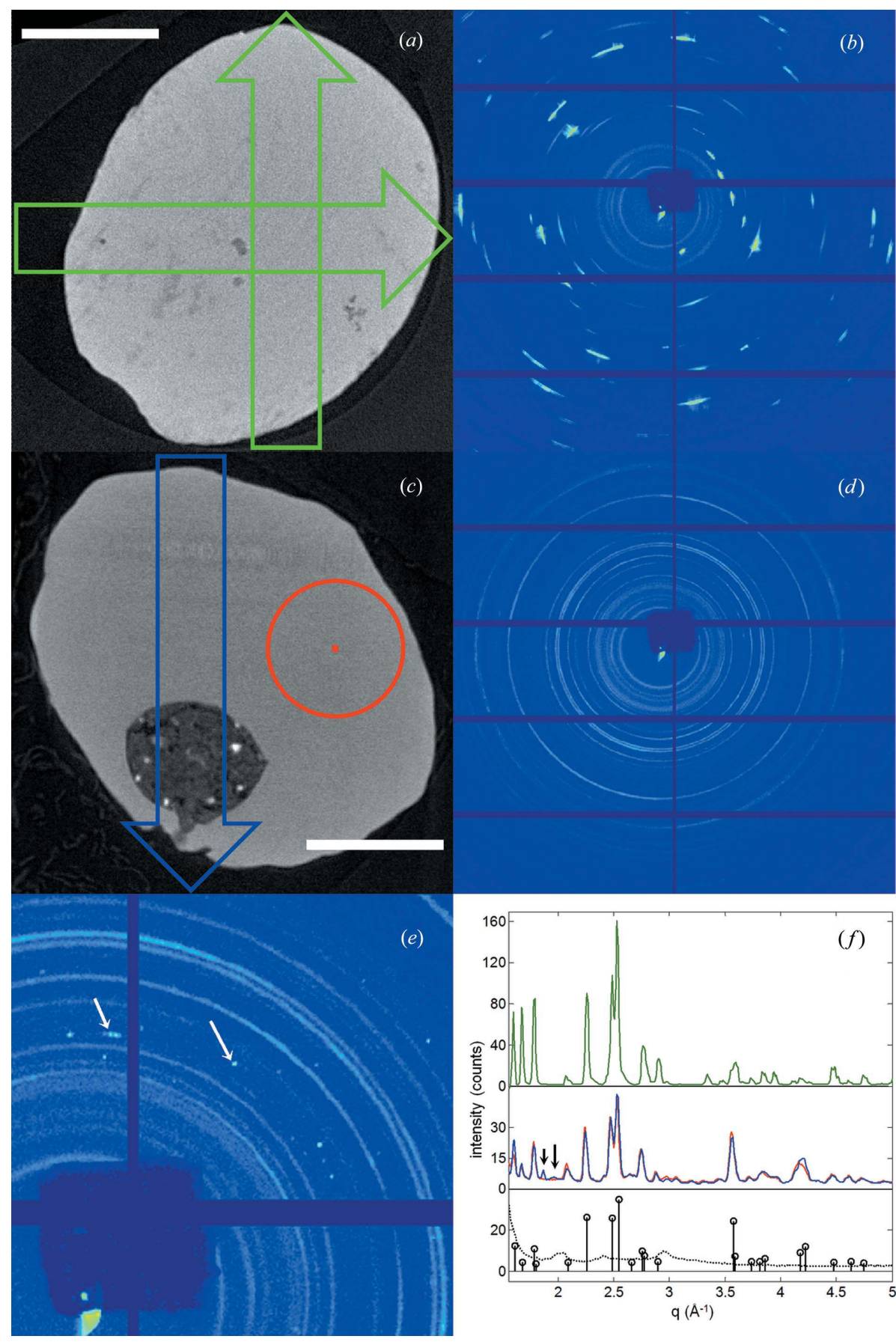

Figure 4

Combined XMT and XRD results on two micrometeorites. (a) XMT reconstruction of sample A, voxel size $0.5 \times$ $0.5 \times 0.5 \mu \mathrm{m}$. Green arrows indicate the beam paths for XRD measurements. Scale bar $200 \mu \mathrm{m}$. $(b)$ Scattering pattern obtained from sample A, corresponding to the horizontal arrow in $(a)$. (c) Vertical slice through the XMT reconstruction of sample $\mathrm{B}$, voxel size $0.5 \times 0.5 \times 0.5 \mu \mathrm{m}$. Markings indicate XRD beam paths, with the beam coming out of the page at the location of the red circle. The dark round object at the bottom is an inclusion of less attenuating material within the micrometeorite. Scale bar $200 \mu \mathrm{m}$. (d) Scattering pattern corresponding to the red circle in $(c)$. The scattering beam was perpendicular to the page, completely missing the inclusion. $(e)$ Scattering pattern corresponding to the blue arrow in $(c)$, where the beam passed through the inclusion in the micrometeorite. White arrows indicate reflections arising from the inclusion. $(f)$ Integrated radial intensity profiles after background subtraction. The horizontal axis is scattering vector magnitude in units of $\AA^{-1}$, and the vertical axes are intensities (integrated counts over $30 \mathrm{~min}$ ). The green line at the top corresponds to sample A (average of the two measurements), the red and blue lines in the middle correspond to panels $(d)$ and $(e)$ (black arrows indicate two diffraction peaks arising from the inclusion), and the dashed black line at the bottom is a typical background intensity from the sample holder. The stem graph shows the expected peak positions and relative intensities of hortonolite, an intermediate member of the solid solution series between olivine minerals fayalite and forsterite (data from Brown \& Prewitt, 1973). For clarity, only the reflections with relative intensity greater than $10 \%$ are shown. spatially localized X-ray scattering experiments. Applicable to a wide range of sample types, it can be used to map the nanoscale structure, i.e. crystal structure information, crystallinity and crystal orientation within the sample, with the aid of a three-dimensional attenuation image of the sample at the micrometre scale. Targeting of the diffraction experiment with $200 \mu \mathrm{m}$ precision is demonstrated by an X-ray diffraction tomography experiment on a silver behenate phantom, and the potential of the system in research applications is illustrated by examining the mineralogical composition of two submillimetre-sized micrometeorites.

The authors would like to express their gratitude to Dr Mika Torkkeli, Mr Pasi Lintunen and Dr Marko Peura for their assistance and discussions in the development of the system, Dr Tomas Kohout of the Division of Geophysics and Astronomy, University of Helsinki, and Dr Jérôme Gattacceca and Dr Pierre Rochette of the University of Aix-Marseill/CNRS for providing the micrometeorite samples and helpful discussions, and Ms Tia Svärd for assistance in constructing the scattering system. This work has been supported by the Academy of Finland (contract No. 1259526). JPS would like to acknowledge The National Doctoral Program for Materials Physics for financial support.

\section{References}

Álvarez-Murga, M., Bleuet, P. \& Hodeau, J.-L. (2012). J. Appl. Cryst. 45, 1109-1124.

Brown, G. E. \& Prewitt, C. T. (1973). Am. Mineral. 58, 577-587.

Brunke, O., Brockdorf, K., Drews, S., Müller, B., Donath, T., Herzen, J. \& Beckmann, F. (2008). Proc. SPIE, 7078, doi:10.1117/12.794789.

Flannery, B. P., Deckman, H. W., Roberge, W. G. \& D'Amico, K. L. (1987). Science, 237, 1439-1444. Huotari, S., Pylkkänen, T., Verbeni, R., Monaco, G. \& Hämäläinen, K. (2011). Nat. Mater. 10, 489-493.

Johnson, G., King, A., Honnicke, M. G., Marrow, J. \& Ludwig, W. (2008). J. Appl. Cryst. 41, 310318.

Kastner, J., Harrer, B., Requena, G. \& Brunke, O. (2010). NDT\&E Int. 43, 599-605.

King, A., Reischig, P., Adrien, J. \& Ludwig, W. (2013). J. Appl. Cryst. 46, 1734-1740.

Leppänen, K., Bjurhager, I., Peura, M., Kallonen, A., Suuronen, J.-P., Penttilä, P., Love, J., Fagerstedt, K. \& Serimaa, R. (2011). Holzforschung, 65, 865-873.

Ludwig, W., Schmidt, S., Lauridsen, E. M. \& Poulsen, H. F. (2008). J. Appl. Cryst. 41, 302309.

Naik, N. N., Jupe, A. C., Stock, S. R., Wilkinson, A. P., Lee, P. L. \& Kurtis, K. E. (2005). Cem. Concr. Res. 36, 144-159.

Nakamura, T., Tsuchiyama, A., Akaki, T., Uesugi, K., Nakano, T., Takeuchi, A., Suzuki, Y. \& 


\section{laboratory notes}

Noguchi, T. (2008). Meteorit. Planet. Sci. 43, 247-259.

Penttilä, P. A., Kilpeläinen, P., Tolonen, L., Suuronen, J.-P., Sixta, H., Willför, S. \& Serimaa, R. (2013). Cellulose, 20, 2335-2347.

Poulsen, H. F. (2012). J. Appl. Cryst. 45, 1084-1097.

Pyzalla, A., Camin, B., Buslaps, T., Di Michiel, M., Kaminski, H., Kottar, A., Pernack, A. \& Reimers, W. (2005). Science, 308, 92-95.

Reischig, P., King, A., Nervo, L., Viganó, N., Guilhem, Y., Palenstijn, W. J., Batenburg, K. J., Preuss, M. \& Ludwig, W. (2013). J. Appl. Cryst. 46, 297-311.
Schroer, C. G., Kuhlmann, M., Roth, S. V., Gehrke, R., Stribeck, N., Almendarez-Camarillo, A. \& Lengeler, B. (2006). Appl. Phys. Lett. 88, 164102.

Stock, S. R. (2008). Int. Mater. Rev. 52, 129-181.

Stock, S. R., Barss, J., Dahl, T., Veis, A. \& Almer, J. D. (2002). J. Struct. Biol. 139, 1-12.

Voltolini, M., Dalconi, M. C., Artioli, G., Parisatto, M., Valentini, L., Russo, V., Bonnin, A. \& Tucoulou, R. (2013). J. Appl. Cryst. 46, 142-152. 